July 1992 [Revised]

DAMTP R-92/24

SNUTP 92-86

\title{
A Master Equation for Multi-Dimensional Non-Linear Field Theories
}

\author{
Q-Han Park \\ Department of Applied Mathematics and Theoretical Physics \\ University of Cambridge, Silver Street, \\ Cambridge, CBз 9EW, U.K. \\ and \\ Department of Physics, Kyung Hee University \\ Seoul, 130-701, Korea
}

\begin{abstract}
A master equation ( $n$ dimensional non-Abelian current conservation law with mutually commuting current components ) is introduced for multi-dimensional non-linear field theories. It is shown that the master equation provides a systematic way to understand 2-d integrable non-linear equations as well as 4-d self-dual equations and, more importantly, their generalizations to higher dimensions.
\end{abstract}

To be published in Physics Letter B

\footnotetext{
${ }^{1}$ Address after Sept.1992; E-mail address; qpark@nms.kyunghee.ac.kr
} 
It is well known that 4-d self-dual equations (both the self-dual Einstein and the self-dual Yang-Mills equations) are integrable via the twistor method ${ }^{[1][2]}$ and that after dimensional reduction, they yield many known integrable non-linear field equations in two spacetime dimensions. ${ }^{[3][4]}$ Unfortunately, higher dimensional $(d \geq 3)$ non-linear field equations, describing non-trivial physical interactions do not arise in this way. Moreover, it is not known whether there exist higher dimensional integrable non-linear field equations similar to the two dimensional ones.

The purpose of this Letter is to introduce a set of simple first order differential equations (the "master equation") and show that it provides a systematic way of obtaining the 4-d self-dual equations as well as generalizing them to higher dimensions (the $2 n$-d Kähler Ricci flat and the Hermitian-Yang-Mills equations). When dimensionally reduced, these higher dimensional "self-dual" equations are shown to become various multi-dimensional non-linear field equations, e.g. the 4-d sine-Gordon and the 4-d non-linear sigma model equations, and it is possible the master equation share the same integrability structure. Thus the master equation provides a simple setting for the investigation of non-linear structures of higher dimensional field equations, and may lead to a deeper understanding of the subject. We discuss the integrability of the master equation and consider the possibility of multi-dimensional integrable field theory.

Consider the following $n$-dimensional equation ("master equation"):

$$
\text { (a) } \nabla \cdot \mathbf{J} \equiv \partial_{i} J_{i}=0 \quad, \quad(b)\left[J_{i}, J_{j}\right]=0 \quad ; \quad i, j=1,2, \ldots, n \quad(n \geq 2)
$$

where $J_{i}$ are components of a vector current $\mathbf{J}$ valued in the Lie algebra $\mathbf{g}$. The bracket denotes a commutator and $\partial_{i} \equiv \partial / \partial x^{i}$ is the partial differentiation with respect to local coordinates $x^{i} ; i=1,2, \ldots, n$. We use the $n$-dimensional Euclidean metric to raise or lower indices. Eq.(1) has a simple interpretation as a current 
conservation law with all the current components mutually commuting. For $n=2$, the master equation becomes integrable and describes a 2-d non-linear sigma model in the following way. Define the dual variable $A_{i} \equiv \epsilon_{i j} J_{j}$, with $\epsilon_{12}=-\epsilon_{21}=1$, to bring eq.(1) into the form

$$
\text { a) } \epsilon_{i j} \partial_{i} A_{j}=0 \quad \text { b) } \quad\left[A_{1}, A_{2}\right]=0 \text {. }
$$

This may be solved partially for $A_{i}=g^{-1} \partial_{i} g$ with $g$ valued in the Lie group $G$. Then the remaining equation becomes

$$
\epsilon_{i j} \partial_{i}\left(g^{-1} \partial_{j} g\right)=0
$$

which is precisely the equation of the 2-d sigma model with a pure Wess-Zumino term. ${ }^{[5]}$ Eq.(2), on the other hand, can be solved completely when we identify it as the integrability condition ( $F_{12}^{\lambda} \equiv\left[L_{1}, L_{2}\right]=0$ ) of the overdetermined linear equations

$$
L_{i} \Phi \equiv\left(\partial_{i}+\lambda A_{i}\right) \Phi=0 \quad ; \quad i=1,2
$$

for any value of $\lambda \in C P^{1}$, and apply the inverse scattering method ${ }^{[6]}$ to the linear equations. In [5], we have shown that the 2 -d master equation $(n=2)$ turns into the 4-d self-dual Einstein and the self-dual Yang-Mills equations when $J_{i}$ take values in the infinite dimensional algebras sdiff $M^{2}$ and $\operatorname{sdiff}_{h} M^{2}$. [ thereby relating 2-d integrable systems with 4-d self-dual systems.

In this Letter, we show that this generalizes to arbitrary $n$ subject to certain subtleties given below. For $n \geq 2$, we assume that $J_{i}$ take values in sdiff $M^{n}$. Let $y^{\mu}(\mu=1, \cdots, n)$ be coordinates on $M^{n}$. We may write $J_{i}$ in a local coordinate

${ }^{2}$ Here sdiff $M^{n}$ denotes the algebra of volume-preserving diffeomorphisms of a manifold $M^{n}$ and $\operatorname{sdif} f_{h} M^{n}$ is its extension by a Lie algebra $\mathbf{h}$. 
$\operatorname{basis}\left\{\partial_{\mu} \equiv \partial / \partial y^{\mu} ; \mu=1,2, \ldots, n\right\}$

$$
J_{i}=\epsilon_{\mu_{1} \cdots \mu_{n}} \partial_{\mu_{2}} V_{i}^{\mu_{3} \cdots \mu_{n}} \partial_{\mu_{1}}
$$

where $V_{i}^{\mu_{1} \cdots \mu_{n-2}}=V_{i}^{\left[\mu_{1} \cdots \mu_{n-2}\right]}$ are functions of the $2 n$ variables $\left(x^{i}, y^{\mu} ; i, \mu=1,2, \ldots, n\right)$. Eq.(1a) can be solved by

$$
V_{i}^{\mu_{3} \cdots \mu_{n}}=\epsilon_{i i_{2} \cdots i_{n}} \partial_{i_{2}} W_{i_{3} \cdots i_{n}}^{\mu_{3} \cdots \mu_{n}}
$$

with arbitrary functions $W_{i_{3} \cdots i_{n}}^{\mu_{3} \cdots \mu_{n}}=W_{\left[i_{3} \cdots i_{n}\right]}^{\left[\mu_{3} \cdots \mu_{n}\right]}$. Note that eq.(5) is invariant under the change $W_{i_{3} \cdots i_{n}}^{\mu_{3} \cdots \mu_{n}} \rightarrow W_{i_{3} \cdots i_{n}}^{\mu_{3} \cdots \mu_{n}}+\partial_{\left[i_{3}\right.} F_{\left.i_{4} \cdots i_{n}\right]}^{\mu_{3} \cdots \mu_{n}}$ or $W_{i_{3} \cdots i_{n}}^{\mu_{3} \cdots \mu_{n}} \rightarrow W_{i_{3} \cdots i_{n}}^{\mu_{3} \cdots \mu_{n}}+\partial^{\left[\mu_{3}\right.} G_{i_{3} \cdots i_{n}}^{\left.\mu_{4} \cdots \mu_{n}\right]}$ for arbitrary $F$ and $G$. This shows that the $J_{i}$ are parameterized by $(n-1)^{2}$ independent variables while the number of remaining equations in eq.(1b) is $\frac{1}{2} n^{2}(n-1)$. Therefore the master equation is overdetermined in general. A particular solution arises if we solve eq.(1) partially in terms of a scalar function $\Omega\left(x^{i}, y^{\mu}\right)$ such that

$$
J_{i}=\epsilon_{\mu_{1} \cdots \mu_{n}} \epsilon_{i i_{2} \cdots i_{n}} \Omega_{, \mu_{2} i_{2}} \cdots \Omega_{, \mu_{n} i_{n}} \partial_{\mu_{1}}
$$

where subscripts with a comma denote partial differentiation. The remaining equations then become

$$
\left[J_{i}, J_{j}\right]=\epsilon_{\mu_{1} \cdots \mu_{n}} \epsilon_{i j i_{3} \cdots i_{n}} \Omega_{, \mu_{3} i_{3}} \cdots \Omega_{, \mu_{n} i_{n}} D_{, \mu_{2}} \partial_{\mu_{1}}=0
$$

where $D$ is the determinant of the $n \times n$ matrix whose $(i, \mu)$ entry is $\Omega_{, i \mu}$. Eq.(8) is satisfied if and only if $D_{, \mu}=\partial_{y^{\mu}} D=0$ for all $y^{\mu}$. Therefore, after the integration, the master equation reduces to

$$
D=\epsilon_{\mu_{1} \cdots \mu_{n}} \epsilon_{i_{1} i_{2} \cdots i_{n}} \Omega_{,_{1} i_{1}} \Omega_{, \mu_{2} i_{2}} \cdots \Omega_{\mu_{n} i_{n}}=\phi\left(x^{1}, \cdots x^{n}\right)
$$

\footnotetext{
${ }^{3}$ We avoid possible global problems and assume that $M^{n}$ is simply connected and vector fields satisfy suitable regularity conditions.
} 
for an arbitrary function $\phi$. In the case $\phi \neq 0, \phi$ can be set to one after a reparametrization of coordinates:

$$
\left(x^{i}, y^{\mu}\right) \rightarrow\left(\tilde{x^{i}}, \tilde{y^{\mu}}\right) ; \quad \partial \tilde{x^{1}} / \partial x^{1}=\phi, \quad \tilde{x^{i}}=x^{i} ; 2 \leq i \leq n, \quad \tilde{y^{\mu}}=y^{\mu} ; 1 \leq \mu \leq n
$$

For $n=2$, eq.(7) is a general solution of eq.(1a) and so eq.(9) is in fact equivalent to the master equation. However, for $n \geq 3$, it is not clear whether eq.(9) is equivalent to the master equation or a special case of it requiring further restrictions. This is an interesting open question which will be pursued elsewhere. Here, we only wish to point out that eq.(9) arises from the master equation and that eq.(9) has a simple geometrical interpretation which generalizes that given in [5] for the $n=2$ case. This is as follows. Consider a Kähler metric given in terms of a potential $\Omega$ :

$$
d s^{2}=g_{a b} d w^{a} d w^{b}=2 \Omega_{, i \mu} d x^{i} d y^{\mu}
$$

Then, eq.(9) with $\phi=1$ means that the determinant of the Kähler metric is constant or, equivalently, that the Ricci tensor vanishes identically, i.e. $R_{a b}=0$. Thus we have shown that the $2 n$-dimensional Kähler Ricci flat equation arises from eq.(1) with the infinite gauge symmetry $\operatorname{sdiff} M^{n}$. For $n=2$, this is equivalent to the 4 -d self-dual Einstein equation while the linear equation (eq.(4)) determines the corresponding twistor space. ${ }^{[1][2]}$ More generally, we may define a Lie algebra $\mathbf{h}$-extension of sdif $f M^{n}$ by the following exact sequence:

$$
0 \rightarrow \mathbf{h} \rightarrow \operatorname{sdiff} f_{h} M^{n} \rightarrow \operatorname{sdiff} M^{n} \rightarrow 0
$$

The algebra sdif $f_{h} M^{n}$ could be represented in terms of "covariantized" volume preserving vector fields such that the $J_{i}$ taking value in sdif $f_{h} M^{n}$ are given by

$$
J_{i}=\epsilon_{\mu_{1} \cdots \mu_{n}} \partial_{\mu_{2}} V_{i}^{\mu_{3} \cdots \mu_{n}} \partial_{\mu_{1}}+K_{i}
$$

\footnotetext{
${ }^{4}$ We regard $\left(x^{i}, y^{\mu}\right)$ as $2 n$ independent complex variables so that we are really considering a complexified Kähler structure. The usual Kähler metric can be recovered by taking $y^{1}=\bar{x}^{1}$ etc. We also use $w^{a}$ to denote the $2 n$ coordinates $\left(x^{i}, y^{\mu}\right)$.
} 
where $K_{i}=K_{i}\left(x^{k}, y^{\mu}\right)$ are arbitrary functions valued in the Lie algebra $\mathbf{h}$. It is easy to check that these covariantized volume preserving vector fields close under commutation and indeed form an algebra. A partial solution of eq.(1) may once again be obtained in terms of a scalar function $\Omega$ satisfying eq.(9) and a holonomy element $U$, valued in the Lie group $\mathrm{H}$ associated with $\mathbf{h}$, namely

$$
J_{i}=\epsilon_{\mu_{1} \cdots \mu_{n}} \epsilon_{i i_{2} \cdots i_{n}} \Omega_{, \mu_{2} i_{2}} \cdots \Omega_{, \mu_{n} i_{n}}\left(\partial_{\mu_{1}}+U^{-1} \partial_{\mu_{1}} U\right)=\frac{g^{i \mu}}{\sqrt{g}}\left(\partial_{\mu}+U^{-1} \partial_{\mu} U\right)
$$

where $U$ satisfies

$$
\epsilon_{\mu_{1} \cdots \mu_{n}} \epsilon_{i i_{2} \cdots i_{n}}\left(\Omega_{, \mu_{2} i_{2}} \cdots \Omega_{, \mu_{n} i_{n}} U^{-1} \partial_{\mu_{1}} U\right)_{, i}=\frac{g^{i \mu}}{\sqrt{g}} \partial_{i}\left(U^{-1} \partial_{\mu} U\right)=0
$$

Here $g^{a b}$ is the inverse of the metric given in eq.(11) and $g$ is the determinant of the metric. For $n=2$, this is precisely Yang's expression for the 4-d self-dual Yang-Mills equation on a self-dual space. ${ }^{[5]}$ Thus eq.(15) generalizes the 4-d self-dual Yang-Mills equation to the higher dimensional one known as the 2n-d Hermitian-Yang-Mills equation:追

$$
\begin{aligned}
F_{i j} & =\left[\frac{\partial}{\partial x^{i}}+A_{x^{i}}, \frac{\partial}{\partial x^{j}}+A_{x^{j}}\right]=0 \\
F_{\mu \nu} & =\left[\frac{\partial}{\partial y^{\mu}}+A_{y^{\mu}}, \frac{\partial}{\partial y^{\nu}}+A_{y^{\nu}}\right]=0 \\
g^{i \mu} F_{i \mu} & =g^{i \mu}\left[\frac{\partial}{\partial x^{i}}+A_{x^{i}}, \frac{\partial}{\partial y^{\mu}}+A_{y^{\mu}}\right]=0
\end{aligned}
$$

which can be shown to be equivalent to eq.(15) by solving eq.(16) for $A_{x^{i}}=P^{-1} \partial_{x^{i}} P$, $A_{y^{\mu}}=Q^{-1} \partial_{y^{\mu}} Q$ and rewriting eq.(17) in terms of $U \equiv Q P^{-1}$. Therefore, we have shown that the master equation systematically generalizes the 4-d self-dual equations to higher dimensions.

\footnotetext{
${ }^{5}$ In general, Hermitian-Yang-Mills connections are given by eq.(16) and eq.(17) but with $g^{i \mu} F_{i \mu}$ being a constant multiple of the identity. Here we take the constant to be zero.
} 
Our identification of the Kähler Ricci flat and the Hermitian-Yang-Mills equations as the proper higher-dimensional generalization of 4-d self-dual equations among other possibilities $^{[7][8]}$ stems from the following three observations, Firstly, they can be expressed as "generalized self-duality conditions" in $2 n$ dimensions: ${ }^{[10]}$ |

$$
\begin{aligned}
R_{a b c d} & ={ }^{*} R_{a b c d} \equiv \frac{1}{2} \sqrt{g} \epsilon_{a b e f i_{1} \mu_{1} \cdots i_{n-2} \mu_{n-2}} g^{i_{1} \mu_{1}} \cdots g^{i_{n-2} \mu_{n-2}} R_{c d} \text { ef } \\
F_{a b} & ={ }^{*} F_{a b} \equiv \frac{1}{2} \sqrt{g} \epsilon_{a b c d i_{1} \mu_{1} \cdots i_{n-2} \mu_{n-2}} g^{i_{1} \mu_{1}} \cdots g^{i_{n-2} \mu_{n-2}} F^{c d}
\end{aligned}
$$

where $\epsilon_{a b \cdots c d}$ is the totally antisymmetric rank $2 n$ tensor with $\epsilon_{x^{1} y^{1} \cdots x^{n} y^{n}}=1$. Second, and more importantly, the Kähler Ricci flat and the Hermitian-Yang-Mills equations reduce to many interesting higher dimensional non-linear (interacting) field equations which generalize 2-d integrable field equations. In the following, we give a few examples. Consider eqs.(15)-(17) in the flat background given by $\Omega=x^{1} y^{1}+x^{2} y^{2}+\cdots+x^{n} y^{n}$. If we take $x^{i}= \pm y^{i}$, eq.(15) trivially reduces to the $n$-dimensional non-linear sigma model equation, $\partial_{i}\left(U^{-1} \partial_{i} U\right)=0$ with various signatures. For another example, make the ansatz $A_{x^{n}}=\Psi, A_{y^{n}}=\Psi^{\dagger}$ with $\Psi$ in the adjoint representation and $y^{i}=\bar{x}^{i}(i=1,2, \ldots, n-1)$, where bar denotes complex conjugation, and where all fields are independent of $x^{n}$ and $y^{n}$. Then eqs.(16) and (17) become

$$
\begin{array}{cl}
{\left[D_{x^{i}}, \Psi\right] \equiv\left[\frac{\delta}{\delta x^{i}}+A_{x^{i}}, \Psi\right]=0 \quad, \quad\left[D_{\bar{x}^{i}}, \Psi^{\dagger}\right] \equiv\left[\frac{\delta}{\delta \bar{x}^{i}}+A_{\bar{x}^{i}}, \Psi^{\dagger}\right]=0} \\
\sum_{i=1}^{n-1} F_{x^{i} \bar{x}^{i}}=-\left[\Psi, \Psi^{\dagger}\right] \quad, \quad F_{x^{i} x^{j}}=0=F_{\bar{x}^{i} \bar{x}^{j}}
\end{array}
$$

which imply the $(2 n-2)$-d Yang-Mills-Higgs equations;

$$
\text { a) }\left[D^{a},\left[D_{a}, \Psi\right]\right]=0 \quad \text { b) }\left[D^{a}, F_{a b}\right]=\left[D_{b},\left[\Psi, \Psi^{\dagger}\right]\right] \text {. }
$$

\footnotetext{
${ }^{6}$ Eq.(19) for $n=3,4$ was first considered in [7]
} 
Now, restrict further by taking the following ansatz for $A_{x^{i}}$ and $\Psi:{ }^{[9]}$

$$
A_{x^{i}}=\sum_{\alpha=1}^{r} A_{x^{i}}^{\alpha} h^{\alpha}=-\sum_{\beta=1}^{r}\left(K^{-1}\right)_{\alpha \beta} \partial_{x^{i}} \ln u_{\beta} h^{\alpha} ; A_{\bar{x}^{i}}=-\sum_{\alpha=1}^{r}\left(A_{x^{i}}^{\alpha}\right)^{*} h^{\alpha} ; \Psi=\sum_{\alpha=1}^{r} u_{\alpha} e^{\alpha}
$$

where $h^{\alpha}, e^{\alpha}$ are Cartan-Weyl basis for the algebra satisfying (without sum)

$$
\left[e^{\alpha}, e^{-\alpha^{\prime}}\right]=\delta_{\alpha \alpha^{\prime}} h^{\alpha}, \quad\left[h^{\alpha}, e^{\beta}\right]=K^{\beta \alpha} e^{\beta}, \quad\left[h^{\alpha}, h^{\beta}\right]=0
$$

Here $r$ is the rank of the group and $K$ is the Cartan matrix. With $\phi_{\alpha} \equiv\left|u_{\alpha}\right|^{2}$, eq.(20) becomes the $(2 n-2)$-d Toda equation;

$$
\nabla^{2} \ln \phi_{\alpha}=-\sum_{\beta=1}^{r} K_{\alpha \beta} \phi_{\beta}
$$

where $\nabla^{2}$ is the $(2 n-2)$-d Laplacian. By using the affine Cartan matrix for the KacMoody algebra, this can be generalized to the affine Toda equation. In particular, for the $\operatorname{sl}(2, R)$ Kac-Moody algebra, it becomes the well-known $(2 n-2)$-d sine(sinh)Gordon equation. We may also consider a reduction of the Kähler Ricci flat equation. Besides the trivial reduction to the 4-d self-dual Einstein equation, it also reduces to less trivial, non-self-dual equations. For example, assume a rotational Killing symmetry s.t. $\Omega=\Omega\left(x^{i}, y^{i}, r \equiv x^{n} y^{n}\right)(i=1, \ldots, n-1)$ and make the change of coordinates: $\left(x^{i}, y^{i}, r\right) \rightarrow\left(p^{i}=x^{i}, q^{i}=y^{i}, w=r \partial_{r} \Omega\right)$. This allows us to introduce a new Kähler potential $\Sigma=\Sigma\left(p^{i}, q^{i}, w\right)$ satisfying $\Omega_{, x^{i}}=\Sigma_{, p^{i}}$. The reduced Kähler Ricci flat equation then becomes

$$
\operatorname{det}\left(\Sigma_{, p^{i} q^{j}}\right)=\left(e^{-\Sigma_{, w}}\right)_{, w}
$$

For $n=2$, eq.(25) was identified in [5] with the $s l(\infty)$-Toda equation. However, this is not in general true for $n \geq 3$. If we restrict further by making the following separation of variables:

$$
\Sigma\left(p^{i}, q^{i}, w\right)=w K\left(p^{i}, q^{i}\right)+f(w)
$$


we get the $(2 n-2)$-d Einstein-Kähler equation:

$$
\operatorname{det}\left(g_{p^{i} q^{j}}\right)=\operatorname{det}\left(K_{, p^{i} q^{j}}\right)=e^{\Lambda K}
$$

with a cosmological constant $\Lambda$. These examples show that most of the four-dimensional extension of two-dimensional relativistic integrable equations can be embedded into the Kähler Ricci flat and the Hermitian-Yang-Mills equations by almost the same reduction procedure as the two dimensional case. However, one should not that since for $n \geq 3$ eqs.(9) and (15) are not necessarily equivalent to the master equation with the previously considered infinite dimensional gauge groups, one may still need further specifications for the precise ansätze. Third, we show that the Hermitian-Yang-Mills equations admit exact multi-centered solutions in the particular case where the gauge group is chosen to be $\operatorname{Sl}(2, C)$. Consider the following ansatz for $A$ with a flat background metric and $y^{i}=\bar{x}^{i}(i=1, \cdots, n)$ :

$$
A_{x^{i}}=\left(\begin{array}{cc}
-\frac{1}{2} \partial_{i} \ln \phi & -\partial_{i} \ln \phi \\
0 & \frac{1}{2} \partial_{i} \ln \phi
\end{array}\right) \quad, \quad A_{y^{\mu}}=\left(\begin{array}{cc}
\frac{1}{2} \partial_{\mu} \ln \phi & 0 \\
-\partial_{\mu} \ln \phi & -\frac{1}{2} \partial_{\mu} \ln \phi
\end{array}\right) .
$$

Then, the (complexified) Hermitian-Yang-Mills equations reduce to

$$
\frac{1}{\phi}\left(\partial_{x^{1}} \partial_{\overline{x^{1}}}+\cdots+\partial_{x^{n}} \partial_{\bar{x}^{n}}\right) \phi=\frac{1}{\phi} \nabla^{2} \phi=0
$$

where $\nabla^{2}$ is the $2 n$-d Laplacian. This may be compared with the 4 -d $S U(2)$ selfdual Yang-Mills case where multi-centered exact solutions with $5 k$ parameters were obtained by reducing the non-linear self-dual Yang-Mills equation to the linear 4-d Laplace equation. ${ }^{[11]}$ However, it should be emphasized that our ansatz is different from those in [11] and does not apply to the $S U(2)$ case, and moreover, makes the Euclidean Yang-Mills action vanish identically. The explicit form of the solutions and further details will be given in [10]. Unfortunately, we have not succeeded in obtaining exact multi-centered solutions for the $S U(2)$ case and other Hermitian 
cases. Nevertheless, there exists a theorem proven by Uhlenbeck and Yau ${ }^{[12]}$ which shows that the moduli space of $2 n$-d Hermitian-Yang-Mills connections is identical to that of stable holomorphic vector bundles over a compact Kähler manifold. This theorem generalizes the theorem of Donaldson for the $n=2$ case $^{[13]}$ which simplifies the ADHM construction ${ }^{[14]}$ of Yang-Mills instantons. Even though the Uhlenbeck and Yau's theorem is not as strong as the ADHM construction to allow the explicit construction of solutions (it is only an existence proof), it provides a certain algebraic setting for the problem and supports our identification of the Hermitian-Yang-Mills equation as the proper higher dimensional generalization of the 4-d self-dual YangMills equation.

Having shown that the master equation implies various multi-dimensional nonlinear field equations which become integrable in two spacetime dimensions, we are now led to the question as to whether these non-linear equations are, in general, integrable. In the $n=2$ case, both the master equation and 4 -d self-dual equations (as well as their 2-d reductions) possess the same integrability structure, i.e. they all appear to be the integrability conditions of the associated linear systems (eq.(3)), while general solutions are obtained by solving the Riemann-Hilbert problem corresponding to linear systems. ${ }^{[1][2][6]}$ This leads us to conjecture that the integrability structure of the Kähler Ricci flat and the Hermitian-Yang-Mills equations as well as their various reductions is essentially the same as that of the master equation. At present, it is not known whether the master equation for $n \geq 3$ is integrable. If so, it would possibly require a new notion of integrability (e.g. flatness associated with the motion of extended objects) and an introduction of higher order non-linear cohomology which would eventually lead to multi- dimensional integrable field theory. This issue will be 
considered elsewhere. ${ }^{[10]}$

\section{ACKNOWLEDGEMENTS}

I would like to thank M.F.Atiyah, N.J.Hitchin for explaining their unpublished work and initiating my interest in 6-d gauge theories. I am also indebted to G.Gibbons, P.Goddard, P.K.Townsend, P.S.Howe, M.Blencowe and S.Majid for discussions and T.Samols for his reading of the manuscript. I am grateful to CTP at Seoul National University, RIMS at Kyoto University for their help during my visit, and above all to S.Hawking and K.Soh for their encouragement.

\section{REFERENCES}

[1] R.Penrose, Gen.Rel.Grav.7 (1976) 31

[2] R.S.Ward,Phys.Lett.61A (1977) 81; M.F.Atiyah and R.S.Ward, Commun.Math.Phys.55, (1977) 117

[3] R.S.Ward,Phil.Trans.Roy.Soc.Lond. A315 (1985) 451; N.J.Hitchin, Proc.Lond.Math. Soc. 55 (1987) 59

[4] I.M.Krichever and S.P.Novikov, Funkts.Analiz.12(4) (1978) 41; L.J.Mason and G.A.J.Sparling, Phys.Lett.137A (1989) 29; I.Bakas and D.A.Depireux, Mod.Phys.Lett. A6 399(1991)

[5] Q-H.Park, Phys.Lett.238B,287 (1990); Phys.Lett.257B, 105(1991); Int.J.Mod.Phys.A, Vol. 7 ,No. 7 (1992) 1415

[6] C.S.Gardner,I.M.Greene,M.D.Kruskal and R.M.Miura, Phys.Rev.Lett. 19 (1967) 1095; V.E.Zakharov and A.B.Shabat, Funct.Anal. and Appl. 13 (1979) 13; V.E.Zakharov and A.V.Mihailov, Sov.Phys.JETP 47 (1978) 1017

[7] E.Corrigan,C.Devchand,D.B.Fairlie and J.Nuyts, Nucl.Phys.B214 (1983) 452; R.S.ward, Nucl.Phys. B236 (1984) 381 
[8] D.H.Tchrakian, J.Math.Phys. 21 (1980) 166

[9] A.N.Leznov and M.V.Saveliev, Commun.Math.Phys. 74 (1980) 111

[10] Q-H. Park, "On Multi-Dimensional Integrable Field Theory", in preparation

[11] G.t'Hooft (unpublished); R.Jackiw, C.Nohl and C.Rebbi, Phys.Rev.D15, (1977) 1642

[12] K.K.Uhlenbeck and S.-T. Yau, Commun.Pure.Appl.Math.39(1986) 257; correction, ibid $\mathbf{4 2 , 7 0 3}$

[13] S.K.Donaldson, Proc.Lond.Math.Soc.3(1985) 1

[14] M.F.Atiyah, V.G.Drinfeld, N.J.Hitchin and Y.I.Manin, Phys.Lett.A65 (1978) 185 